\title{
A call to action: why medical education curriculum needs to encourage young physicians to innovate
}

\author{
Aditi A. Sharma ${ }^{1}$ (D) Kachiu C. Lee ${ }^{2} \cdot$ Lilit Garibyan $^{3}$
}

Received: 8 September 2020 / Revised: 23 November 2020 / Accepted: 7 December 2020 / Published online: 12 January 2021

(c) The Author(s), under exclusive licence to Springer-Verlag GmbH, DE part of Springer Nature 2021

\begin{abstract}
Patient-centered and physician-led innovations are key to promoting physicians as visionary leaders in the healthcare system especially during times of crises. COVID-19 has inspired some promising recent advancements within medicine worth noting, including improvements in telemedicine, 3-D printed personal protective equipment (PPE) and ventilators, drug and vaccine development, sterilization of PPE allowing for reuse, and point of care testing; they highlight a broader lesson for how we might innovate better within medicine, even after the crisis has passed. As such, with the complexities of modernday medicine, to continue to foster this culture of innovation, it is paramount that going forward, medical education adapt and embrace an innovation curriculum that prepares physicians and healthcare workers to work with their communities and researchers to confidently tackle any challenges that may present. Integrating innovation into our careers and medical training is important for advancement of the field and to be able to handle challenges that may present to the healthcare system.
\end{abstract}

Keywords Innovation $\cdot$ Medical education $\cdot$ Entrepreneur $\cdot$ COVID-19

COVID-19 has shocked the world and caught many countries unprepared, halted travel and daily work rituals, challenged the economy, and forced nations to improvise how best to protect the health of their citizens. Despite all the confusion and uncertainty, the power of individuals and communities to innovate shows what impressive feats in a short time can be achieved when people are united by necessity. Some promising recent advancements within medicine are worth noting, including the improvements in telemedicine, 3-D printed personal protective equipment (PPE) and ventilators, drug and vaccine development, sterilization of PPE allowing for reuse, and point of care testing; they highlight a broader lesson for how we might innovate better within medicine, even after the crisis has passed. As such, with the complexities of modern-day medicine, to continue

Lilit Garibyan

lgaribyan@mgh.harvard.edu

1 Department of Dermatology, University of California, Irvine, 118 Med Surge I, Irvine, CA 92697, United States

2 Main Line Center for Laser Surgery, Philadephia, USA

3 Department of Dermatology, Harvard Medical School, Wellman Center for Photomedicine, Massachusetts General Hospital, 50 Blossom Street-Thier 2, Boston, MA 02114, USA to foster this culture of innovation, it is paramount that going forward, medical school education adapt and embrace an innovation curriculum that prepares physicians and healthcare workers to quickly identify unmet medical needs, and work with their communities and researchers to bring new solutions to solve those problems.

Current medical education focuses on the rapid acquisition and application of preexisting knowledge. Trainees are assessed on skill procurement and the demonstration of content expertise-e.g., what are the causes of ST-elevation on an ECG? What are the 12 cranial nerves and their functions? Please interpret a chest X-ray. This paradigm yields essential skills, as physicians are taught standard of care. What current medical education lacks, however, is an emphasis on problem and need-based innovation that leads to new solution creation. A foundation in design thinking framework at a time of crisis could be critical in saving time and lives by bringing timely solutions to help physicians and patients.

Clinicians have unique insight into medical problems and unmet needs as they face patient-related problems daily. In addition, clinicians are the end-users of the technology invented to solve patient's unmet medical needs. These two factors combined give clinicians the ability to play a critical role in biomedical innovation. Historically, innovation in medical training was essential due to necessity, and as 
such, an important skill of being a physician. Most notably, Dr. Edward Jenner invented the first vaccine for smallpox, after realizing people who received small aliquots of the infection gained immunity, thereby saving millions of lives and ultimately leading to the eradication of smallpox worldwide [1]. Similarly, in order to understand the systems of the body through minimally invasive methods, Nobellaureate and physician Dr. Willem Einthoven invented the ECG to characterize the electrical currents of the heart, and the gynecologist, Dr. Karl Theo Dussik, pioneered the first use of the ultrasound [2].

So, how do we engage the current generation of young physicians in biomedical innovation? This is multifactorial, but we believe a starting point is addressing the more recent disconnect in medical education and innovation, given the emphasis of current medical school education on skill procurement and content expertise.

\section{Innovation skills should be taught early}

How to identify and solve unmet needs through innovation training should begin early in medical school and continue longitudinally through residency and beyond. In our prior survey study, over $60 \%$ of physicians had no training in innovation, although the majority wanted more training [3]. The innovation curriculum should emphasize the goal of cultivating patient-centered innovation where clinicians identify problems worth solving, practice divergent and convergent brainstorming, collaborate with stakeholders, prototype and commercialize solutions. Throughout this journey of innovation, learners should have the opportunity to partner with other expert clinicians and non-physicians (e.g., scientists, engineers) to select which patient problems are worth solving and what solutions are worthy of further development and testing [4]. Early introduction to this problem-based innovation design process could be extremely helpful in mobilizing physicians to help identify problems and solutions quickly during a crisis, such as a pandemic. One such program, the Magic Wand Initiative, has been created at Harvard Medical School, and successfully implemented a clinician-driven program to cultivate patient-centered innovation at Massachusetts General Hospital Department of Dermatology [5]. The outcomes from this program have clearly demonstrated that when clinicians are educated and empowered to identify and solve problems, new and innovative solutions are brought to clinic to improve patient care [6]. Blake et al. showed through a review and thematic analysis that there were thirteen medical education programs in the USA participating in Innovation and Entrepreneurship curriculums [7]. Furthermore, Cohen et al. showed that the return on investment of Innovation and Entrepreneurship programs on the undergraduate level at the University of
Michigan resulted in 18 novel projects with patent filings, three schoolwide shark tank competitions and 6 start-up companies [8]. Ultimately, expanding similar training programs will empower clinicians with the skillsets to easily identify and convey unmet medical needs to non-clinicians ${ }^{4}$, especially in times of need.

\section{Interdisciplinary collaborations need to be nurtured}

In addition, we believe that institutions and communities should promote interdisciplinary resources to allow for collaboration among physicians and non-physicians such as engineers, scientists, business managers, to provide an optimized environment for innovation. Riita Katila et al. at Stanford reviewed 231 medical-device start-ups over 25 years. They noted successful innovation for medical-device startups is heavily dependent on participation of doctors because of their unique insight on medical problems and developed technologies but concluded that many doctors do not have the training or skills to manage the broader process of innovation. Thus, the ability to integrate multidisciplinary teams to include those with physicians, engineers, and business managers are critical to making medical innovation come to fruition [9].

During COVID-19, the potential of implanting web-based education has been explored in a very short time, and this ability to connect through web-based platforms could be harnessed going forward to connect medical students with non-physicians as well as leaders in innovation, to increase access to education regarding innovation and entrepreneurship [10]. Integration of medical students and residents during this viral pandemic may accelerate the continuing transformation away from traditional teaching methods of approaching medicine [11] and foster innovative solutions to critical problems through interdisciplinary collaboration.

\section{Collaboration at the national and international levels should be promoted}

Finally, participation in innovation opportunities within medicine through conferences, fellowship opportunities, Hackathons [4], Shark Tank-like sessions at the national and international level should be encouraged to promote the next generation of physician innovators. The access to these national and international opportunities will connect innovators from all over the world, allowing individuals with a wide array of talents and ideas to come together and innovate the best solutions.

Programs like the Virtual Magic Wand program, run by physicians at Massachusetts General Hospital, are making 
inroads toward training physician innovators. Scholars in prior cohorts have gone on to start their own start-up companies focused on developing a more effective solution for wart treatment in children, innovative solutions to the PPE shortage, cordless hyfrecators, smartphone apps used for taking standardized photographs over time, and repurposing existing medications for field cancerization.

COVID-19 has undoubtedly changed the way medicine will be practiced in the future. Embracing innovation in medical school education could change the future response to medical crises. This begins with exposure to biomedical device innovation, collaboration, and a focus on developing programs that support this process at the community, institutional, national, and international levels in order to empower physicians to address any challenges they may encounter in medicine.

Hopefully, the changes in attitudes, mindsets, and energies that are uniting people now will persist once this emergency is over. We firmly believe that a shift, from the current medical paradigm of needing to "know everything," to one that embraces and develops a framework on how to identify problems and develop solutions to meet unmet needs, will significantly empower the healthcare workforce to better handle any challenges that may present.

\section{Compliance with ethical standards}

Conflicts of interest The authors declare that they have no conflict of interest.

\section{References}

1. Smallpox vaccines [Internet]. World Health Organization. 2017 [cited 2020 Apr 15]; Available from: https://www.who.int/csr/ disease/smallpox/vaccines/en/
2. Med School Consulting: MDconsultants [Internet]. MDconsultants why physicians need to innovate comments. [cited $2020 \mathrm{Apr}$ 15]. Available from: https://mdconsultants.ca/why-physicians -need-to-innovate/

3. Lee KC, Lee I, Okhovat J et al (2020) Innovation interest within dermatology: a needs assessment for novel thought processes. Arch Dermatol Res. https://doi.org/10.1007/s00403-020-02118-6

4. Costa A, Duerksen L, Raff A, Xu S. The case for hackathons in dermatology [Internet]. Dermatology Times. 2019 [cited 2020 Apr 15]. Available from: https://www.dermatologytimes.com/ innovation/case-hackathons-dermatology

5. Garibyan L, Anderson RR (2017) Increasing clinical faculty engagement in problem-driven research. JAMA Dermatology 153(5):375

6. Garibyan L, Kroshinsky D, Freeman E, Sakamoto FH, Anderson RR (2020) A strategy for empowering clinicians and increasing innovation: the magic wand initiative. Arch Dermatol Res. https ://doi.org/10.1007/s00403-020-02111-z

7. Niccum BA, Sarker A, Wolf SJ, Trowbridge MJ (2017) Innovation and entrepreneurship programs in US medical education: a landscape review and thematic analysis. Medical Education Online 22:1. https://doi.org/10.1080/10872981.2017.1360722

8. Cohen MS (2017) Enhancing surgical innovation through a specialized medical school pathway of excellence in innovation and entrepreneurship: lessons learned and opportunities for the future. Surgery 162(5):989-993. https://doi.org/10.1016/j. surg.2017.06.012

9. Katila R, Thatchenkery S, Christensen MQ, Zenios S (2017) Is there a doctor in the house? Expert product users, organizational roles, and innovation. Acad Manag J 60(6):2415-2437

10. Woolliscroft JO (2020) Innovation in response to the COVID19 pandemic crisis. Acad Med 95(8):1140-1142. https://doi. org/10.1097/ACM.0000000000003402

11. McMaster D, Veremu M, Santucci C (2020) COVID-19: opportunities for professional development and disruptive innovation. Clin Teach 17(3):238-240. https://doi.org/10.1111/tct.13175

Publisher's Note Springer Nature remains neutral with regard to jurisdictional claims in published maps and institutional affiliations. 